\title{
Factores asociados a la depresión en estudiantes foráneos de Medicina, Universidad Católica de Cuenca, 2019
}

\section{Factors associated with depression in foreign medical students, Universidad Católica de Cuenca, 2019}

\author{
DOI: $10.46932 / s f j d v 3 n 1-069$
}

Received in: Jan 30st, 2021

Accepted in: Feb 1th, 2022

\section{Cinthia Gabriela Romero Sarango}

Médico General egresada de la Universidad de Cuenca - Ecuador

Machala - El Oro - Ecuador

E-mail: gabriela.romero92@ucuenca.edu.ec

Michell Susan Saavedra Palacios

Médico General egresada de la Universidad de Cuenca - Ecuador

Cuenca - Azuay - Ecuador

E-mail: michellesaavp@ hotmail.com

\section{Tania Diciana Arévalo Córdova}

Médico General egresada de la Universidad Técnica de Machala - Ecuador

Machala-El Oro - Ecuador

E-mail: tarevalo_est@utmachala.edu.ec

Mishell Katherine Zurita Peña

Médico General egresada de la Universidad Técnica de Machala - Ecuador

Universidad Técnica de Machala

Machala - El Oro - Ecuador

E-mail: mishell_1607@hotmail.com

\section{Amy Andrea Armijos Dutan}

Médico General egresada de la Universidad Espíritu Santo - Ecuador

Machala - El Oro - Ecuador

E-mail: Amyarmijosd@gmail.com

\section{RESUMEN}

Introducción: La depresión, se caracteriza por un conjunto de síntomas físicos, emocionales, cognitivos y conductuales; que afecta notablemente la vida de las personas, principalmente en el rango de 20 - 45 años. Objetivo: Determinar los factores asociados a la depresión en estudiantes foráneos de la carrera de medicina de la Universidad Católica de Cuenca. Metodología: Estudio cuantitativo, descriptivo y transversal. La población estuvo constituida por los estudiantes foráneos de la carrera de medicina de la Universidad Católica de Cuenca, Provincia del Azuay, de 1ero a 10mo semestre del periodo lectivo Marzo - Julio de 2019. Para la asociación de la depresión y los factores de riesgo, se usó el Odds Ratio. Resultados: La población de estudio estaba en el rango de 18 a 30 años, con una media de 21 años DE \pm 2 años, con prevalencia en el rango de 18 a 21 años (51.6\%) y el sexo femenino con un 62,6\%. La prevalencia de depresión fue de $62.6 \%$. Los factores de riesgo tuvieron asociación con la depresión fueron: el sexo femenino (OR: 2,60 IC 95\%: 1,39 - 4,86 p= 0,002); tener poco o ningún apoyo social 
(OR: 9,56 IC 95\%: 0,54 -170,06 $\mathrm{p}=0,04)$; y no tener pareja civil (OR: 4,25 IC 95\%: 1,06 - 17,01 $\mathrm{p}=$ 0,03), todos con significancia estadística. Conclusión: Existe alta prevalencia de depresión entre los estudiantes foráneos de la Universidad Católica de Cuenca. Los factores de riesgo que tuvieron asociación con la depresión fueron: el sexo femenino, el poco apoyo social y no tener pareja civil

Palabras clave: Depresión, estudiante de medicina, estudiantes foráneos, factores asociados, factores de riesgo.

\begin{abstract}
Introduction: Depression is characterized by a set of physical, emotional, cognitive and behavioral symptoms; that notably affects the lives of people, mainly in the range of 20 - 45 years. Objective: To determine the factors associated with depression in foreign students of the medical career of the Catholic University of Cuenca. Methodology: Quantitative, descriptive and cross-sectional study. The population was constituted by foreign students of the medical career of the 7 Catholic University of Cuenca, Province of Azuay, from 1st to 10th semester of the school period March - July 2019. For the association of depression and risk factors, Odds Ratio was used. Results: The study population was in the range of 18 to 30 years, with a mean of 21 years $\mathrm{SD} \pm 2$ years, with a prevalence in the range of 18 to 21 years $(51.6 \%)$ and the female sex with $62.6 \%$. The prevalence of depression was $62.6 \%$. The risk factors associated with depression were: female sex (OR: $2.6095 \% \mathrm{CI}$ : 1.39 - $4.86 \mathrm{p}=0.002)$; having little or no social support (OR: 9.56 95\% CI: $0.54-170.06 \mathrm{p}=0.04$ ); and not having a civil partner (OR: 4.25 95\% CI: 1.06 - 17.01 $\mathrm{p}=0.03$ ), all with statistical significance. Conclusion: There is a high prevalence of depression among foreign students of the Catholic University of Cuenca. The risk factors that were associated with depression were: female sex, little social support and not having a civil partner.
\end{abstract}

Keywords: Depression, medical student, foreign students, associated factors, risk factors.

\title{
1 INTRODUCCIÓN
}

La depresión, es una enfermedad que se caracteriza por un estado de abatimiento, sentimientos de tristeza, que causan alteraciones de comportamiento como: un pérdida de interés, del placer, disminución de la energía, sentimientos de culpa o baja estima, alteración del sueño, apetito y falta de concentración ( Vargas et al, 2017; Rodríguez-Castro, et al. 2018).

Es uno de los trastornos mentales más comunes, con un aproximado de 280 millones de personas en el mundo que padecen depresión, convirtiéndose en un grave problema de salud, especialmente si la depresión es recurrente y de intensidad moderada a grave. Esta enfermedad puede causar sufrimiento a la persona afectada y alterar sus actividades laborales, escolares y familiares, llevando en el peor de los casos al suicidio (OMS, 2021).

La prevalencia mundial del trastorno depresivo varía del 8 al 25\% en la población, con una relación mujer - hombre de 2 a 1, con promedio de edad entre 20 y 45 años (Organización Mundial de la Salud OMS, 2001). Se considera que contribuye significativamente a la carga mundial de morbilidad y afecta a personas de todos los países del mundo con una prevalencia global de episodios depresivos del 3,2\%. 
Estos trastornos depresivos generalmente comienzan a una edad temprana y son recurrentes a lo largo de la vida (Cui R, 2016; Ngasa et al. 2017).

Según el Ministerio de Salud Pública (2022), en el 2015, en las atenciones ambulatorias, el número de casos reportados de depresión fue tres veces mayor en las mujeres que en los hombres. Registrando en ese año 50.379 personas con diagnóstico presuntivo y definitivo por depresión; de las cuales, 36.631 corresponden a mujeres y 13.748 a hombres, con 3406 hospitalizaciones relacionadas con casos de depresión.

Se considera que la prevalencia de los trastornos mentales es más frecuente en poblaciones con características particulares. Entre las poblaciones que se han analizado se encuentran los estudiantes de la carrera de medicina, debido a las altas exigencias sociales, psicológicas y académicas que demanda esta carrera. Dentro de los factores a los que se encuentran expuestos está la excesiva carga de trabajo, las pérdidas académicas, la falta de apoyo económico, problemas de adaptación a otra ciudad, entre otros (Roman et al, 2008, Dyrbye, et al. 2005; Benbassat et al, 2011; Gonzalez-Olaya et al. 2014) .

Por estos antecedentes, el objetivo del presente estudio es determinar los factores asociados a la depresión en estudiantes foráneos de la carrera de medicina de la Universidad Católica de Cuenca.

\section{MATERIALES Y MÉTODOS}

Se realizó un estudio cuantitativo, descriptivo y transversal. La población estuvo constituida por los estudiantes foráneos de la carrera de medicina de la Universidad Católica de Cuenca, Provincia del Azuay, de primero a décimo semestre del periodo lectivo Marzo - Julio de 2019.

Se realizó la evaluación del apoyo social mediante la escala de apoyo social (AFA-R15) y la evaluación de la depresión se realizó mediante el test de Hamilton. Para la asociación de la depresión y los factores asociados, los datos fueron dicotomizados y como medida de asociación se usó el Odds Ratio.

Para la realización del estudio, se obtuvo el permiso del Decano de la Facultad de Medicina de la Universidad Católica de Cuenca, Dra. Susana Peña y el consentimiento informado de los informados, previo a la aplicación del instrumento de recolección de datos y aplicación de la escala de apoyo social y test de Hamilton. Para el análisis estadístico se usó el software IBM Statistical Package for the Social Sciences (SPSS) versión 26.

\section{RESULTADOS}

La población estuvo constituida por 182 estudiantes de medicina foráneos, en un rango de 18 a 30 años, con una media de 21 años DE \pm 2 años, siendo el sexo prevalente el femenino con 114 estudiantes (Figura 1). El rango de edad prevalente fue de 18 a 21 años con un $51.6 \%$, siendo la mayoría de estudiantes 
de estado civil soltero, con un $94 \%$ y el semestre con mayor número de estudiantes foráneo fue de 7 a 8 semestre (Tabla 1).

Figura 1 Edad de estudiantes de medicina foráneos

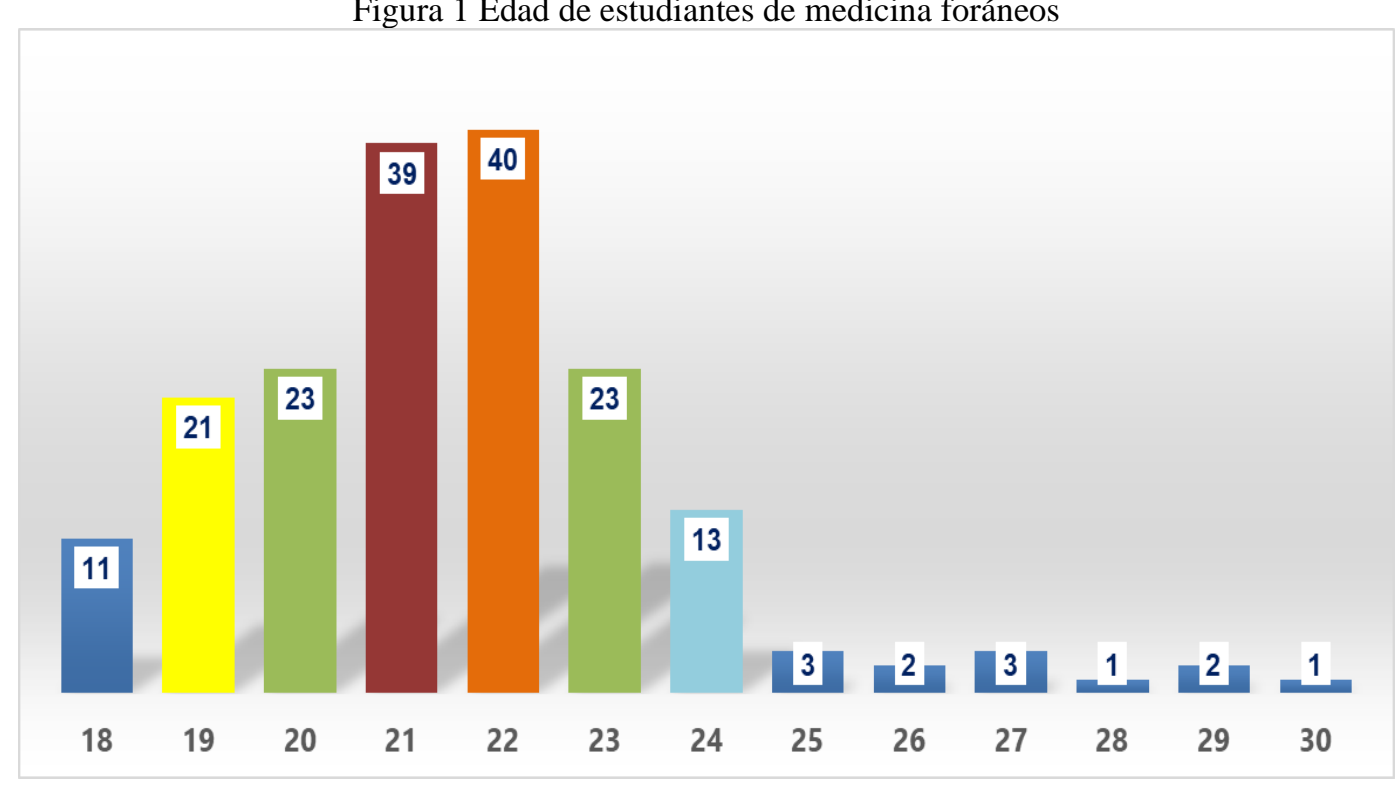

Tabla 1. Datos sociodemográficos de los estudiantes foráneos Universidad Católica de Cuenca

\begin{tabular}{|c|c|c|c|c|c|c|c|}
\hline \multirow{2}{*}{\multicolumn{2}{|c|}{ Variable }} & \multicolumn{2}{|c|}{$\begin{array}{c}\text { Masculino } \\
(n=68)\end{array}$} & \multicolumn{2}{|c|}{$\begin{array}{c}\text { Femenino } \\
(n=114)\end{array}$} & \multicolumn{2}{|c|}{$\begin{array}{c}\text { Total } \\
(\mathbf{n}=\mathbf{1 8 2})\end{array}$} \\
\hline & & f & $\%$ & f & $\%$ & f & $\%$ \\
\hline \multicolumn{8}{|l|}{ Rango de edad } \\
\hline & $18-21$ & 33 & $48,5 \%$ & 61 & $53,5 \%$ & 94 & $51,6 \%$ \\
\hline & $22-25$ & 30 & $44,1 \%$ & 49 & $43,0 \%$ & 79 & $43,4 \%$ \\
\hline & $26-29$ & 4 & $5,9 \%$ & 4 & $3,5 \%$ & 8 & $4,4 \%$ \\
\hline & $\geq 30$ & 1 & $1,5 \%$ & 0 & $0,0 \%$ & 1 & $0,5 \%$ \\
\hline \multicolumn{8}{|l|}{ Estado Civil } \\
\hline & casado/a & 3 & $4,4 \%$ & 4 & $3,5 \%$ & 7 & $3,8 \%$ \\
\hline & unión libre & 0 & $0,0 \%$ & 3 & $2,6 \%$ & 3 & $1,6 \%$ \\
\hline & soltero/a & 64 & $94,1 \%$ & 107 & $93,9 \%$ & 171 & $94,0 \%$ \\
\hline & divorciado/a & 1 & $1,5 \%$ & 0 & $0,0 \%$ & 1 & $0,5 \%$ \\
\hline \multicolumn{8}{|c|}{ Semestre que cursa } \\
\hline & 1-2 semestre & 15 & $22,1 \%$ & 23 & $20,2 \%$ & 38 & $20,9 \%$ \\
\hline & 3-4 semestre & 17 & $25,0 \%$ & 22 & $19,3 \%$ & 39 & $21,4 \%$ \\
\hline & 5-6 semestre & 10 & $14,7 \%$ & 16 & $14,0 \%$ & 26 & $14,3 \%$ \\
\hline & $7-8$ semestre & 21 & $30,9 \%$ & 37 & $32,5 \%$ & 58 & $31,9 \%$ \\
\hline & 9-10 semestre & 5 & $7,4 \%$ & 16 & $14,0 \%$ & 21 & $11,5 \%$ \\
\hline
\end{tabular}

La prevalencia de depresión en estudiantes foráneos, fue de 62.6\%, de 114 las mujeres que participaron en el estudio, 81 presentaron depresión (71.1\%), mientras que de 68 varones, 33 presentaron depresión (48.5\%) siendo el sexo femenino el de mayor prevalencia de depresión. En relación a la evaluación de la depresión, el $27.5 \%$ presentaron depresión leve, el 16.5\% moderada y el 9.3\% grave y severa, respectivamente. 
Tabla 2 Prevalencia de Depresión en Estudiantes foráneos

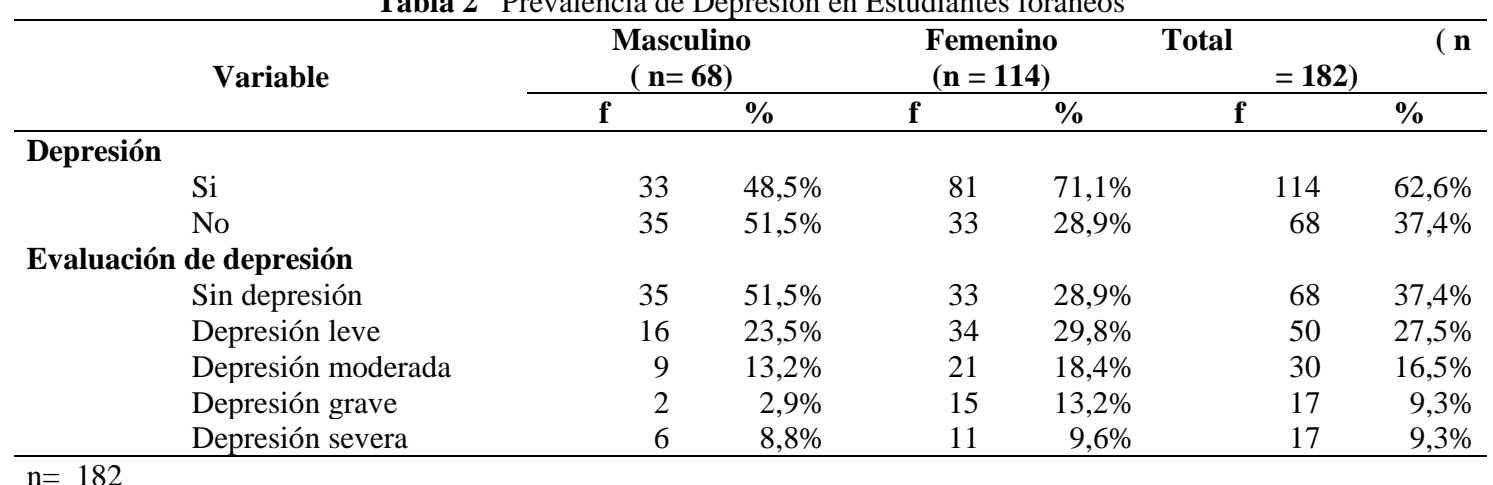

En relación a los factores que se asocian a la depresión, tenemos al sexo femenino, con un OR: 2,60 (IC 95\%: 1,39 - 4,86 $p=0,002$ ); tener poco o ningún apoyo social OR: 9,56 IC 95\%: 0,54 a170,06 $p=0,04$ ); $y$ no tener pareja civil OR: 4,25 (IC 95\%: 1,06 - 17,01 $p=0,03$ ), todos con significancia estadística. El hecho de no sentirse satisfecho con la carrera que se encuentra estudiando, tuvo asociación con la depresión OR: 8,21 (IC 95\%: 0,45 - 148,02 $p=0,15$ ), pero sin significancia estadística.

Por su parte, los factores que tuvieron una leve asociación con la depresión, sin significancia estadística fueron: la edad menor a 24 años OR: 1,38 (IC 95\%: 0,59 - 3,24 $p=0,46$ ); el tener familia monoparental OR: 1,77 (IC 95\%: 0,88 - 3,55 p=0,19); cursar los últimos 2 años de la carrera OR: 1,51 (IC 95\%: 0,28 - 8,03 p=0,63); autofinanciarse los estudios OR: 1,51 (IC 95\%: 0,286 - 8,03 $p=0,62$ ); y usar drogas OR: 1,81 (IC 95\%: 0,66- 4,98 p=0,25).

Los factores que tuvieron asociación protectora, aunque sin significancia estadística, fueron: el vivir acompañado OR: 0,88 (IC 95\%: 0,47 -1,67 $p=0,70$ ); y una carga horaria menor a 8 horas OR: 0,61 (IC 95\%: 0,33 - 1,13 $p=0,12$ ) (tabla 3). 
Tabla 3. Asociación entre factores familiares y depresión

\begin{tabular}{|c|c|c|c|c|c|}
\hline \multirow[t]{2}{*}{ Variable } & \multicolumn{2}{|c|}{$\begin{array}{c}\text { Con } \\
\text { depresión } \\
(\mathbf{n}=114)\end{array}$} & \multicolumn{2}{|c|}{$\begin{array}{c}\text { Sin depresión } \\
\quad(n=68)\end{array}$} & \multirow{2}{*}{$\begin{array}{c}\text { Odss Ratio } \\
\text { IC 95\% } \\
\end{array}$} \\
\hline & f & $\%$ & f & $\%$ & \\
\hline \multicolumn{6}{|l|}{ Sexo } \\
\hline Femenino & 81 & $44,5 \%$ & 33 & $18,1 \%$ & \multirow{2}{*}{$\begin{array}{l}\text { OR: 2,60 (IC: } 1,39- \\
4,86) \quad p=0,002\end{array}$} \\
\hline Masculino & 33 & $18,1 \%$ & 35 & $19,2 \%$ & \\
\hline \multicolumn{6}{|r|}{ 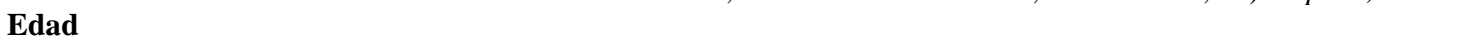 } \\
\hline 18 a 23 años & 100 & $54,9 \%$ & 57 & $31,3 \%$ & \multirow{2}{*}{$\begin{array}{c}\text { OR: } 1,38 \text { (IC: } 0,59-3,24) \\
p=0,46\end{array}$} \\
\hline$\geq 24$ años & 14 & $7,7 \%$ & 11 & $6,0 \%$ & \\
\hline \multicolumn{6}{|l|}{ Estado Civil } \\
\hline Sin Pareja civil & 111 & $61,0 \%$ & 61 & $33,5 \%$ & \multirow{2}{*}{$\begin{array}{ll}\text { OR: } 4,25 & \text { (IC: } 1,06- \\
17,01) & p=0,02\end{array}$} \\
\hline Con Pareja civil & 3 & $1,6 \%$ & 7 & $3,8 \%$ & \\
\hline \multicolumn{6}{|l|}{ Tipo de familia } \\
\hline Monoparental & 38 & $20,9 \%$ & 15 & $8,2 \%$ & \multirow{2}{*}{ 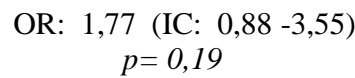 } \\
\hline Biparental & 76 & $41,8 \%$ & 53 & $29,1 \%$ & \\
\hline \multicolumn{6}{|l|}{ Con quien vive } \\
\hline Solo & 37 & $20,3 \%$ & 24 & $13,2 \%$ & \multirow{2}{*}{$\begin{array}{cl}\text { OR: } 0,88 & \text { (IC: } 0,47- \\
1,67) & p=0,70\end{array}$} \\
\hline Acompañado & 77 & $42,3 \%$ & 44 & $24,2 \%$ & \\
\hline \multicolumn{6}{|l|}{ Año que cursa } \\
\hline 3 primeros años & 66 & $36,3 \%$ & 37 & $20,3 \%$ & \multirow{2}{*}{$\begin{array}{ll}\text { OR: } 1,51 & (\text { IC: } 0,28- \\
8,03) & p=0,63\end{array}$} \\
\hline 2 últimos años & 48 & $26,4 \%$ & 31 & $17,0 \%$ & \\
\hline \multicolumn{6}{|l|}{ Satisfacción con la carrera } \\
\hline Poco o nada satisfecho & 6 & $3,3 \%$ & 0 & $0,0 \%$ & \multirow{2}{*}{$\begin{array}{l}\text { OR: } 8,21 \text { (IC: } 0,46- \\
148,02) \quad p=0,15\end{array}$} \\
\hline Moderada - muy satisfecho & 108 & $59,3 \%$ & 68 & $37,4 \%$ & \\
\hline \multicolumn{6}{|l|}{ Carga horaria } \\
\hline$>8$ horas & 37 & $20,3 \%$ & 30 & $16,5 \%$ & \multirow{2}{*}{$\begin{array}{c}\text { OR: } 0,61 \text { (IC: } 0,33- \\
1,130) \quad p=0,12\end{array}$} \\
\hline$<8$ horas & 77 & $42,3 \%$ & 38 & $20,9 \%$ & \\
\hline \multicolumn{6}{|l|}{ Apoyo social } \\
\hline Poco o ninguno & 7 & $3,8 \%$ & 0 & $0,0 \%$ & \multirow{2}{*}{$\begin{array}{c}\text { OR: } 9,56 \quad(0,54 \mathrm{a}-170,06) \\
p=0,04\end{array}$} \\
\hline Si recibe & 107 & $58,8 \%$ & 68 & $37,4 \%$ & \\
\hline \multicolumn{6}{|l|}{ Financiamiento } \\
\hline Autofinanciado & 5 & $2,7 \%$ & 2 & $1,1 \%$ & \multirow{2}{*}{$\begin{array}{l}\text { OR: } 1,51 \text { (IC: } 0,286- \\
8,03) p=0,62\end{array}$} \\
\hline Financiado por terceros & 109 & $59,9 \%$ & 66 & $36,3 \%$ & \\
\hline \multicolumn{6}{|l|}{ Consumo de drogas } \\
\hline $\mathrm{Si}$ & 10 & $5,5 \%$ & 9 & $4,9 \%$ & \multirow{2}{*}{$\begin{array}{c}\text { OR: } 1,81 \text { (IC: } 0,66-4,98) \\
p=0,25\end{array}$} \\
\hline No & 30 & $16,5 \%$ & 49 & $26,9 \%$ & \\
\hline
\end{tabular}

\section{DISCUSIÓN}

En el presente estudio, la prevalencia en los estudiantes foráneos fue muy elevada, con un $62.6 \%$, en contraposición con los estudio realizados por Bernita et al (2017), en el cual los estudiantes de medicina de la Universidad Católica de Cuenca - Ecuador, presentó el 47.7\% de depresión; en el Estudio realizado por Chapilliquen R. realizado en Perú, en estudiantes Universitarios foráneos de la Universidad Privada Antenor Orrego, Trujillo- Perú, en donde la prevalencia fue de 22.91\%; y, el estudio realizado por Martínez M., en estudiantes universitarios de la carrea de Medicina, de la Universidad de Puebla México, donde la depresión fue de $31.72 \%$.

El sexo femenino fue el de mayor prevalencia en depresión con un 71,1\%, teniendo el sexo femenino asociación con la depresión, con un OR: 2,60 (IC 95\%: 1,39-4,86 p=0,002), en concordancia con el estudio realizado por Rodríguez (2018), en el cual el 57.55\% tenían depresión y el estudio realizado por Obregon et al. (2020), en donde el sexo femenino presentó un factor de riesgo de 1,03 veces más para padecer depresión. 


\section{REFERENCIAS}

Bernita E., Castillo F. y Vallejo E. (27 de julio de 2017) Prevalencia de depresión y factores asociados en los estudiantes de Medicina de la Universidad Católica de Cuenca. 2014. Rev Fac Cienc Médicas Univ Cuenca; 35(1):68-73.

Disponible

en: https://publicaciones.ucuenca.edu.ec/ojs/index.php/medicina/article/view/1240/1078

Benbassat J, Baumal R, Chan S, Nirel N. (2005) Sources of distress during medical training and clinical practice: suggestions for reducing their impact. Med Teach, 33: 486-90.

Cui R. (2015) Editorial (Selección temática: una revisión sistemática de la depresión), Neurofarmacología actual, 13(4). Disponible en: https://dx.doi.org/10.2174/1570159X1304150831123535

Dyrbye LN, Thomas MR, Shanafelt TD. Medical student distress: causes, consequences, and proposed solutions. Mayo Clin Proc. 2005 Dec;80(12):1613-22. Available in: https://dx.doi.org/10.4065/80.12.1613

González-Olaya, H., Delgado-Rico, H., Escobar-Sánchez, M. y Cárdenas- Angelone, M. (2014). Asociación entre el estrés, el riesgo de depresión y el rendimiento académico en estudiantes de los primeros semestres de un programa colombiano de medicina. FEM: Revista de la Fundación Educación Médica, 17(1), 47-54. Disponible en: https://dx.doi.org/10.4321/S2014-98322014000100008

Jacobs G. (2002) Non academic factors affecting the academic success of Grenadian students at St. George's University. St. George's: University Centre; 2002

Martínez-Martínez M del C, Muñoz-Zurita G, Rojas-Valderrama K, Sánchez-Hernández JA. Prevalencia de síntomas depresivos en estudiantes de la licenciatura en Medicina de Puebla, México. Aten Fam. 1 de octubre de 2016;23(4):145- $149 . \quad$ Disponible en: https://www.sciencedirect.com/science/article/pii/S140588711630150X

Ministerio de Salud Pública (26 de enero de 2022). Este 7 de abril se celebra el Día Mundial de la Salud, con el tema "Depresión: Hablemos". Noticias Destacadas. Disponible en: https://www.salud.gob.ec/este7-de-abril-se-celebra-el-dia-mundial-de-la-salud-con-el-tema-depresion-hablemos/

Ngasa, SN, Sama, CB., Dzekem, BS et al. (2017). Prevalencia y factores asociados con la depresión entre estudiantes de medicina en Camerún: un estudio transversal. BMC Psiquiatría 17, 216. Disponible en: https://doi.org/10.1186/s12888-017-1382-3

Obregón-Morales, Berea, Montalván-Romero, José C., Segama-Fabian, Edinho, Dámaso-Mata, Bernardo, Panduro-Correa, Vicky, \& Arteaga-Livias, Kovy. (2020). Factors associated with depression in medical students from a peruvian university. Educación Médica Superior, 34(2), e1881. Epub 01 de junio de 2020. Recuperado en 27 de enero de 2022, de http://scielo.sld.cu/scielo.php?script=sci_arttext\&pid=S0864-21412020000200013\&lng=es\&tlng=en.

Organización Mundial de la Salud OMS. (13 de Septiembre de 2021) Depresión. Centro de prensa. Disponible en: https://www.who.int/es/news-room/fact-sheets/detail/depression

Organización Mundial de la Salud. (2001) Informe sobre la salud en el mundo 2001: Salud mental : nuevos conocimientos, nuevas esperanzas. Organización Mundial de la Salud. Disponible en: https://apps.who.int/iris/handle/10665/42447 
Ríos Chapilliquen, E. J. (2018) Antecedente de migración como factor asociado a depresión en estudiantes del primer año de medicina. Disponible en: http://repositorio.upao.edu.pe/handle/20.500.12759/4008

Rodríguez-Castro AI, Ríos-González CM.(2018) Frecuencia de depresión en estudiantes de medicina.Medicina Clínica y Social, 2018;2(3):128-135. Disponible en: https://www.medicinaclinicaysocial.org/index.php/MCS/article/view/60/57

Vargas M, et al. (2017) Influencia de los hábitos en la depresión del estudiante de medicina peruano: estudio en siete departamentos. Rev Colomb Psiquiat. Disponible en: http://dx.doi.org/10.1016/j.rcp.2017.01.008 